Artículo original

\title{
Fractura osteocondral abierta del penacho de la falange distal en punta de dedo en niños: una lesión frecuentemente no diagnosticada
}

\author{
Open osteochondral fracture of the distal phalanx tuft in young children's \\ fingertip: a frequently non-diagnosed injury
}

\author{
Ricardo Kaempf de Oliveira' João Pedro Farina Brunelli² Márcio Aita ${ }^{3}$ Pedro J. Delgado 4 \\ Enrique Vergara-Amador ${ }^{5}$ \\ ' Santa Casa de Misericórdia de Porto Alegre, Porto Alegre, Brasil. \\ ${ }^{2}$ Hospital Santa Casa de São Paulo, São Paulo, Brasil. \\ Centro Universitário Faculdade de Medicina do ABC, Santo André, Brasil. \\ ${ }^{4}$ Hospital Universitario HM Montepríncipe, Boadilla del Monte, Madrid, España. \\ ${ }^{5}$ Universidad Nacional de Colombia, Unidad de Ortopedia y Traumatología, Bogotá D.C, Colombia. \\ Correspondencia: Ricardo Kaempf de Oliveira. Santa Casa de Misericórdia de Porto Alegre. Porto Alegre. Brasil. \\ Correo electrónico: ricardokaempf@gmail.com.
}

\section{Información del artículo Resumen}

Recibido: $10 / 11 / 2020$

Aceptado: 05/03/2021

Palabras clave: traumatismo de los dedos; lesiones de la punta del dedo; uñas; matriz ungueal; traumatismos de las manos.

Keywords: finger injuries; fingertip injuries; nails, hand injuries, nail bed lacerations.

DOI: https://doi.org/10.25214/28056272.1180
La mano es la región del cuerpo más afectada por los traumatismos en los niños. Los más pequeños a menudo sufren traumas por aplastamiento en la punta del dedo, lesiones cuyo tratamiento recibe poca atención en el servicio de urgencias y que muchas veces deja secuelas importantes.

En los menores de 4 años, la epífisis proximal de la falange distal no está calcificada, por lo que no es visible en las radiografías, y la porción calcificada es mucho más pequeña. Al comparar con niños mayores, en este grupo de edad la punta del dedo está formada y sostenida más por tejidos blandos y cartílago, ya que la falange está cubierta en toda su superficie por un casquete condral. Por tal razón, durante una lesión por aplastamiento con flexión de la porción distal de la punta del dedo, la región más frágil es la unión osteocondral del penacho de la falange. Esto provoca lesión con desprendimiento de la placa ungueal, lesión de la matriz ungueal estéril, laceración de la piel con hueso expuesto y fractura osteocondral.

El objetivo del presente estudio fue describir, mediante el reporte de dos casos, el mecanismo y el tratamiento de la lesión por aplastamiento de la punta del dedo en niños pequeños con fractura osteocondral expuesta asociada a daño en la matriz ungueal. Este tipo de lesiones requieren mayor atención en urgencias y deben tratarse como una fractura abierta para reducir el riesgo de infección y secuelas funcionales.

\footnotetext{
Abstract

The hand is the region of the body most affected by trauma in children. Crushing trauma to the fingertip often occurs in young children. Often, in the emergency department, little importance is given to the correct treatment of these injuries, often leaving important sequelae. In young children, less than four years of age, the proximal epiphysis of the distal phalanx is not calcified, so it is not visible on radiographs. Also, the calcified portion of the phalanx is much smaller. In this age group, proportionally, compared to older children, the fingertip is formed and supported more by soft tissues (pulp) and by cartilage, since the phalanx is covered over its entire surface by a chondral cap. Thus, at this stage, during a crush injury, with flexion of the distal portion of the fingertip (a mechanism like Seymour's fracture), the most fragile region is the osteochondral junction of the phalanx tuft. This results in a detached nail plate injury, sterile nail matrix injury, and skin laceration with exposed bone and osteochondral fracture. Our objective is to describe this type of fingertip crush injury in young children, with exposed osteochondral fracture associated with damage to the nail matrix, and his treatment. This injury is an open fracture that compromises the distal osteochondral junction, behaving very similarly to the Seymour fracture that is located proximally in the phalanx. We want to draw attention to the fact that the generalized idea that injuries in children evolve well, despite the severity and regardless of the treatment carried out, is wrong in many cases.
} 


\section{Introducción}

La inmadurez de la coordinación motora en los niños, asociada a su necesidad de aprender a través de la exploración del entorno, hace que las puntas de sus dedos sean un sitio frecuente de trauma por aplastamiento ${ }^{1-4}$. Estas lesiones son más comunes en niños menores de 5 años y generalmente se producen en el hogar o la escuela al cerrar abruptamente puertas, cajones y ventanas ${ }^{5-8}$.

La gravedad de las lesiones por aplastamiento de la punta de los dedos en niños (APDN) varía mucho, al igual que su tratamiento. Las lesiones complejas con pérdida de sustancia (amputación) y las fracturas abiertas requieren cirugía. Por otro lado, las laceraciones -que pueden estar asociadas con lesiones neurológicas y tendinosas o lesiones del aparato ungueal — pueden tratarse de forma conservadora o quirúrgica ${ }^{9-13}$.

La complejidad de la anatomía de la punta de los dedos y la participación de diferentes estructuras anatómicas generan confusión sobre el tratamiento correcto de estas lesiones. La falta de consenso en la literatura ocasiona que muchos cirujanos se sientan incómodos en el tratamiento de niños con estas heridas, ya que el riesgo de complicaciones - como deformidades óseas y ungueales, rigidez e infección- no es bajo ${ }^{5,8,14}$.

La dificultad para definir el tratamiento aumenta cuando se consulta la literatura, ya que los diferentes tipos de lesión son ubicados en un mismo cluster. Un metaanálisis sobre este tema no pudo mostrar diferencias en el tiempo de curación, la tasa de infección y la necrosis de la piel entre los niños que fueron tratados mediante cirugía asociada con apósitos en serie con membrana de silicona (apósito malla de silicona) y los que fueron tratados de forma conservadora con apósitos simples. Los diferentes abordajes (conservador o quirúrgico) tampoco mostraron diferencias cuando se analizaron los resultados en el seguimiento tardío en cuanto a función de la punta del dedo, crecimiento y forma de la uña en la serie que no separó las lesiones en subtipos específicos ${ }^{1,8,14,15}$.

El objetivo del presente estudio fue describir un mecanismo no revelado de lesión de APDN con fractura osteocondral abierta asociada a lesión de la matriz ungueal con el fin de brindar una orientación de su tratamiento y evidenciar que la idea de que las lesiones en los niños evolucionan bien a pesar de la gravedad e independientemente del tratamiento realizado no es la regla general.

\section{Historia y descripción de la lesión}

En 1966, Seymour ${ }^{16}$ describió un tipo de lesión de la punta del dedo en niños en la que, por el mecanismo de aplastamiento y flexión de la punta, se genera una fractura expuesta en la región yuxtaepifisaria, con la consecuente lesión de la matriz ungueal estéril y el desplazamiento de la placa ungueal con interposición de la matriz germinal en el foco de la fractura (Figura 1). En la fractura de Seymour, la punta del dedo permanece flexionada con una deformidad similar a la de un dedo en martillo. La deformidad es mantenida por el tendón extensor, que se inserta en la porción dorsal de la epífisis proximal de la falange y deja este segmento alineado o ligeramente extendido, mientras que la diáfisis y la punta del dedo (porción más distal) se flexionan por la acción del flexor profundo ${ }^{17,18}$.
Figura 1. Fractura de Seymour clásica. Fuente: Elaboración propia.

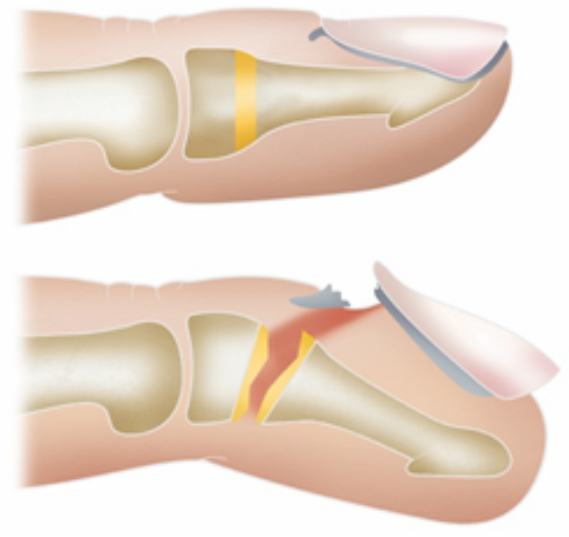

La lesión de Seymour, según su descripción original, se produce en la fisis porque esta es la región más frágil de la falange distal desde los 5 años hasta la adolescencia. En la mayoría de las series, la fractura de Seymour ocurre más entre los 8 y los 10 años $^{17-21}$. Es importante mencionar que Al-Qattan ${ }^{20}$ describió una serie de pacientes con esta lesión que no incluyó a ningún niño menor de 3 años.

Desde el nacimiento hasta alrededor de los 4 años, la epífisis proximal de la falange distal no está calcificada, por lo que la fisis no es visible en las radiografías. Además, la porción calcificada (hueso) de la falange es mucho más pequeña. En comparación con los niños mayores, en este grupo de edad la punta del dedo está formada y soportada más por tejidos blandos (pulpejo) y cartílago que por hueso, ya que la falange está cubierta en toda su superficie por un casquete condral (Figura 2).

Figura 2. Falange en niños menores de 4 años. A: porción calcificada; B: tejidos blandos.

Fuente: Elaboración propia.
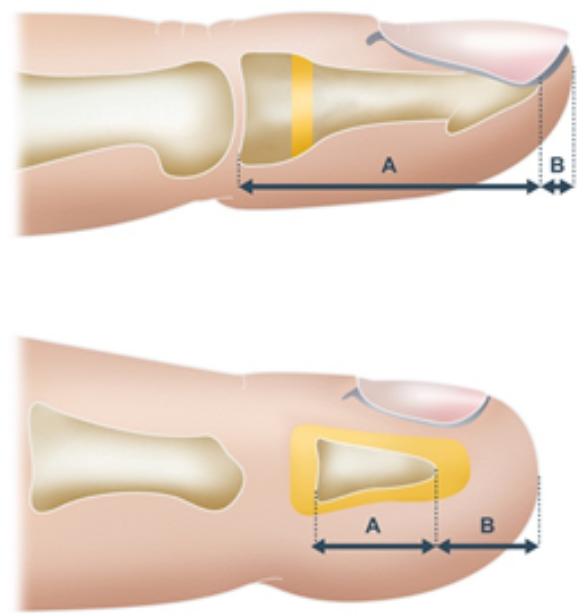
Así, durante una lesión por aplastamiento con flexión de la porción distal de la punta del dedo (mecanismo similar a la fractura de Seymour), en los niños pequeños la región más frágil es la unión osteocondral del penacho de la falange. Esto provoca lesión con desprendimiento de la placa ungueal, lesión de la matriz ungueal estéril, laceración de la piel con exposición ósea y fractura osteocondral (Figura 3). Por tratarse de una lesión en la unión osteocondral y no de tejido óseo calcificado, las radiografías de este tipo de lesión son normales - no muestran fracturas - o, en ocasiones, muestran una avulsión dorsal mínima, lo que confunde el diagnóstico en la urgencia y no muestra la severidad de la lesión. Tal como la fractura de Seymour, esta también es una lesión grave, una fractura abierta.

Figura 3. Fractura osteocondral expuesta por aplastamiento de la punta del dedo en niños pequeños.

Fuente: Elaboración propia.
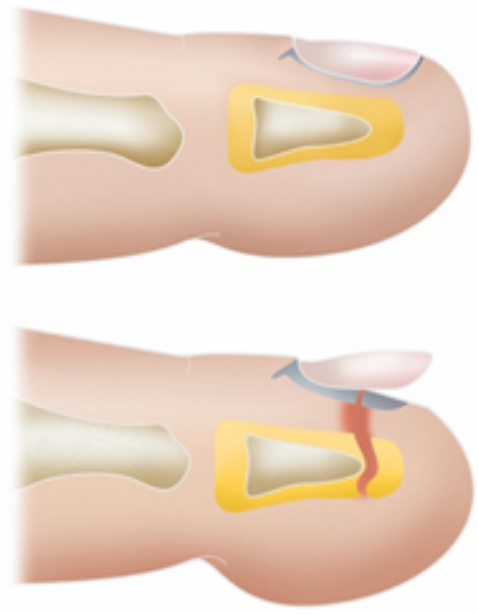

\section{Técnica quirúrgica}

Para las fracturas osteocondrales en lesiones de APDN, el tratamiento debe ser quirúrgico y con anestesia general. Inicialmente, el paciente se coloca en posición supina y se utiliza una mesa de apoyo para las manos y un torniquete neumático en todo el miembro superior o en el dedo.

Después de la antisepsia de rutina, la placa ungueal se retira con cuidado para acceder a la matriz ungueal estéril y al sitio de la fractura. Posteriormente, se realiza un delicado y minucioso desbridamiento con limpieza de huesos y tejidos blandos. El siguiente paso es reducir la fractura osteocondral y fijar con un clavo de Kirschner de $1.0 \mathrm{~mm}$ o $1.2 \mathrm{~mm}$, con control de la reducción y posicionamiento del clavo mediante fluoroscopia. El clavo queda percutáneo, lo que facilita su extracción.

La estabilización de la fractura corrige la deformidad de la punta del dedo, el defecto cutáneo y la lesión de la matriz ungueal, lo cual favorece la sutura. Inicialmente, se sutura la piel alrededor de la matriz con Monocryl ${ }^{\circledR} 4.0$, que permite estabilidad y firmeza en la punta del dedo. A continuación, se realiza la sutura de la matriz estéril con suturas absorbibles (Vicryl ${ }^{\circledR} 6.0$ incoloro) y luego se reemplaza y se fija la placa de la uña.

Posteriormente, se cubre la punta del dedo con gasa antiadherente (Adaptic $\left.{ }^{\circledR}\right)$ y se inmoviliza con una férula de aluminio. Los apósitos se cambian cada semana y el dedo se mantiene inmovilizado durante 4 semanas, tiempo tras el cual los tejidos han curado y se piensa que hay consolidación, por lo que se retira el clavo de Kirschner. La uña tarda un promedio de 6 meses en crecer y lucir estéticamente bien.

\section{Descripción de dos pacientes}

Se trataron dos pacientes con fractura osteocondral abierta en una lesión de APDN; a continuación se describe cada tratamiento y sus resultados.

\section{Caso 1}

Niño de 2 años que sufrió un accidente doméstico al aplastarse la punta del tercer dedo de la mano. Al llegar al servicio de urgencias, la lesión fue diagnosticada como laceración cutánea y deformidad de la punta del dedo (Figuras 4A, 4B y 4C). El equipo de cirugía de la mano constató que se trataba de una fractura osteocondral abierta, aunque tuviera radiografía normal (Figuras 4E y $4 \mathrm{~F}$ ). El paciente fue llevado a cirugía y, tras retirar la placa ungueal, se observó claramente la lesión de la matriz estéril y la fractura osteocondral expuesta (Figura 4D). Previa limpieza y desbridamiento, se realizó estabilización ósea, sutura de la matriz estéril y recambio de la uña (Figura 4G). Después de 4 meses de evolución, el paciente mostró una buena recuperación (Figura $4 \mathrm{H}$ ).

Figura 4. A, B y C) lesión por aplastamiento de la punta del tercer dedo de la mano en paciente de 2 años, con laceración cutánea y deformidad de la punta del dedo; D, E y F) fractura osteocondral abierta con radiografía normal; G) fijación ósea con clavo y sutura de matriz estéril; H: buena recuperación tras 4 meses de evolución.

Fuente: imágenes obtenidas durante la realización del estudio.
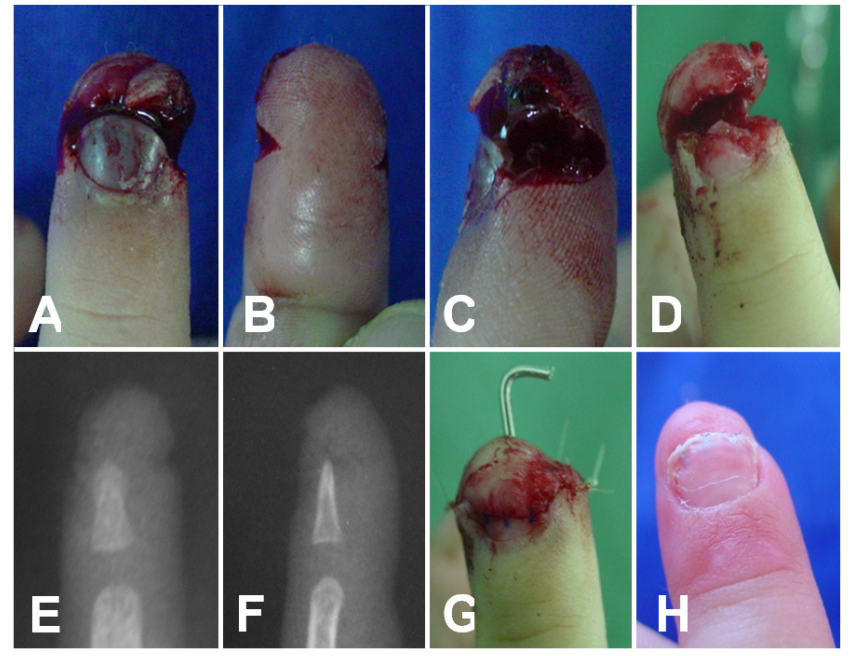

Caso 2

Niña de 4 años que sufrió aplastamiento de las puntas del tercer y el cuarto dedo de la mano derecha con una ventana de su escuela. Ambos dedos presentaban una laceración abierta de la matriz ungueal 
estéril, pero la herida del cuarto dedo era más profunda y provocó un desprendimiento del pulpejo del dedo (Figuras 5A y 5B). Sin embargo, incluso con una deformidad acentuada, las radiografías no mostraron cambios (Figura 5C). El equipo de cirugía de la mano evaluó al paciente y diagnosticó fractura osteocondral abierta. El paciente fue intervenido con limpieza y desbridamiento de la lesión, fijación de la fractura y reparación de la matriz (Figura 5D), tras lo cual presentó una excelente evolución y a los 5 meses la punta del dedo estuvo completamente funcional y estéticamente bien (Figura 5E)

Figura 5. A y B) lesión por aplastamiento del tercer y cuarto dedo derecho en paciente de 4 años, con herida abierta de la matriz ungueal estéril más severa en el cuarto; C) radiografías que no muestran cambios; D) desbridamiento, fijación de la fractura y reparación de la matriz; E) excelente evolución tras 5 meses de posoperatorio.

Fuente: imágenes obtenidas durante la realización del estudio.

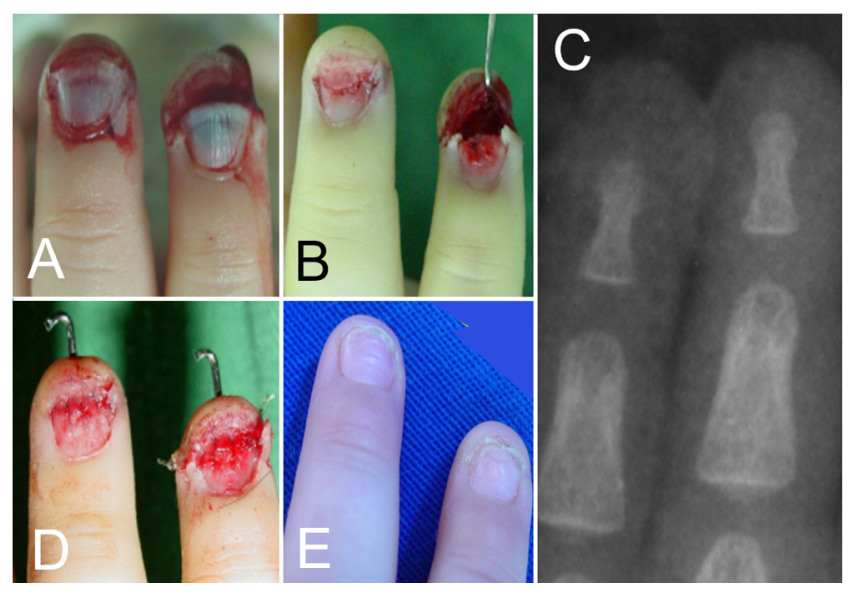

\section{Discusión}

Las fracturas osteocondrales, aunque raras, no son nuevas en los niños. Son lesiones provocadas por traumatismos de alta energía que provocan dolor, incapacidad funcional y deformidad. Se deben considerar las fracturas graves y desviadas para un tratamiento especializado. La mayoría de las fracturas osteocondrales tienen un diagnóstico difícil, requieren una alta sospecha clínica y, a menudo, precisan de tratamiento quirúrgico ${ }^{22}$.

Waters et al. ${ }^{23}$ describieron las lesiones de codo TRASH (The Radiographic Appearance Seemed Harmless Lesions), que inicialmente parecen benignas, pero en realidad son fracturas transfisarias y transcondrales graves; estas ocurren en niños pequeños (menores de 10 años) después de un trauma de alta energía. Algunos ejemplos de lesiones TRASH son: fractura no osificada del cóndilo humeral medial, fractura transfisaria del húmero distal, fractura del epicóndilo medial con interposición intraarticular, fractura por compresión anterior de la cabeza radial y fractura por cizallamiento del cóndilo lateral.

En 2001, Al-Qattan ${ }^{24}$ propuso una clasificación para otro tipo de fractura osteocondral en niños al publicar una serie de fracturas en el cuello de la falange de la mano, un sitio poco frecuente de fracturas en adultos. Por su parte, Tal et al. ${ }^{25}$ señalaron que estas lesiones son más frecuentes en la falange media de los niños, demostraron que el fragmento distal puede presentar diferentes grados de desplazamiento y rotación y evidenciaron que con frecuencia se requiere cirugía para su tratamiento.

Las fracturas de la falange distal representan casi la mitad de los casos de fracturas de la mano y el 50\% de ellas tienen una lesión asociada de la matriz ungueal. Por lo tanto, la mayoría de las lesiones por APDN están relacionadas con fracturas. Además, las fracturas del penacho de la falange distal representan el $80 \%$ de las fracturas de falange en niños menores de 4 años y afectan con mayor frecuencia los dedos largos (tercero y cuarto) ${ }^{1,8}$.

Aunque la mano es la región del cuerpo más afectada por los traumatismos en los niños, sus lesiones aún están poco estudiadas y valoradas. Aunque en los niños pequeños se presentan a menudo traumas por APDN, en los servicios de urgencias se le da poca importancia al correcto tratamiento de estas patologías, que pueden provocar secuelas a largo plazo.

Debido a la existencia de pacientes con lesiones y patologías más graves, no es inusual que las lesiones de las puntas de los dedos sean infravaloradas y muchas veces no sean tratadas por médicos especializados. La correcta valoración en urgencias puede definir la gravedad de la lesión, determinar la necesidad de tratamiento especializado y evitar así las secuelas definitivas. Se ha demostrado que los mejores resultados estéticos y funcionales se obtienen cuando se realiza un correcto tratamiento ${ }^{1,8,9}$.

En 1990, Van Beek et al. ${ }^{26}$ clasificaron las lesiones traumáticas de la matriz ungueal en función del sitio afectado (germinal y matriz estéril), pero no consideraron la edad del paciente. A partir del tratamiento de pacientes pediátricos, los autores observaron que en los niños pequeños la lesión de la matriz estéril a menudo se asocia con una fractura abierta, incluso con radiografías normales, ya que se presenta una pequeña osificación de la falange distal. La laceración de la piel alrededor de la uña y la deformidad de la punta del dedo pueden ser indicadores clínicos de que la lesión presenta una fractura abierta. La lesión osteocondral aquí descrita debe sospecharse en un traumatismo por APDN, incluso cuando se presentan radiografías que no muestran fractura.

En el tratamiento de las lesiones por APDN asociadas a fracturas, la fijación de la fractura y la interfalángica distal no son la regla. Sin embargo, como recomiendan Abzug y Kozin ${ }^{18}$, la estabilización es más adecuada porque brinda mayor comodidad y seguridad en la inmovilización y debido al riesgo de que los niños pequeños no sigan la indicación médica de reposo.

Por su parte, Klifto et al. ${ }^{27}$ llamaron la atención sobre la mayor incidencia de lesiones en las puntas de los dedos en niños que sufren abuso infantil, lo que demuestra que el aplastamiento puede ser parte del abuso o ser causado por descuido al dejar a los niños pequeños sin supervisión.

Las lesiones por APDN también generan un alto grado de estrés para toda la familia, que se siente culpable por la lesión, y un alto costo para el sistema de salud. Por tanto, además del tratamiento correcto, es necesario promover medidas de prevención. Dado que la mayoría de los accidentes ocurren en el hogar o en la escuela, es importante que en estos lugares los niños pequeños sean supervisados constantemente y que se instalen mecanismos de seguridad en puertas, ventanas y cajones ${ }^{1,3}$.

\section{Conclusiones}

Así como la fractura de Seymour es ampliamente conocida y tratada de forma correcta, se debe prestar especial atención a la fractura 
osteocondral de la punta del dedo descrita en este estudio. Existe consenso en que el mejor momento para el tratamiento de las lesiones en la punta del dedo es en la consulta por urgencia. Por tanto, para reducir el riesgo de infección y las secuelas funcionales, este tipo de lesión debe tratarse como una fractura abierta: con cirugía, limpieza especializada, estabilización ósea y reconstrucción del aparato ungueal.

\section{Referencias}

1. Venkatesh A, Khajuria A, Greig A. Management of pediatric distal fingertip injuries: a systematic literature review. Plast Reconstr Surg Glob Open. 2020;8(1):e2595. DOI: 10.1097/ GOX.0000000000002595.

2. Wahba G, Cheung K. Pediatric hand injuries: practical approach for primary care physicians. Can Fam Physician. 2018;64(11):803-10.

3. Yorlets RR, Busa K, Eberlin KR, Raisolsadat MA, Bae DS, Waters PM, et al. Fingertip injuries in children: epidemiology, financial burden, and implications for prevention. Hand (N Y). 2017;12(4):342-7. DOI: 10.1177/1558944716670139.

4. Doraiswamy NV, Baig H. Isolated finger injuries in children--incidence and aetiology. Injury. 2000;31(8):571-3. DOI: 10.1016/ s0020-1383(00)00052-8.

5. Reid S, Duncan MJ. Interventions for treating fingertip entrapment injuries in children. Paediatr Child Health. 2016;21(1):278. DOI: 10.1093/pch/21.1.27.

6. Inglefield CJ, D'Arcangelo M, Kolhe PS. Injuries to the nail bed in childhood. J Hand Surg Br. 1995;20(2):258-61. DOI: 10.1016/s0266-7681(05)80066-x.

7. Ardouin T, Poirier P, Rogez JM. Les traumatismes des extrémités digitales et de l'appareil unguéal chez l'enfant. A propos de 241 cas. Rev Chir Orthop Reparatrice Appar Mot. 1997;83(4):330-4.

8. Al-Qadhi S, Chan KJ, Fong G, Al-Shanteer S, Ratnapalan S. Management of uncomplicated nail bed lacerations presenting to a children's emergency department. Pediatr Emerg Care. 2011;27(5):379-83. DOI: 10.1097/PEC.0b013e318216b22d.

9. Silva JB, Gerhardt S. Trauma to the nail complex. Rev Bras Ortop. 2014;49(2):111-5. DOI: 10.1016/j.rboe.2014.02.005.

10. Papadonikolakis A, Li Z, Smith BP, Koman LA. Fractures of the phalanges and interphalangeal joints in children. Hand Clin. 2006;22(1):11-8. DOI: 10.1016/j.hcl.2005.10.001.

11. Giddins GE, Hill RA. Late diagnosis and treatment of crush injuries of the fingertip in children. Injury. 1998;29(6):447-50. DOI: 10.1016/s0020-1383(98)00083-7.

12. Schultz J, Schröttner P, Leupold S, Dragu A, Sußmann S, Haase $\mathrm{M}$, et al. Conservative treatment of fingertip injuries in children - first experiences with a novel silicone finger cap that enables woundfluid analysis. GMS Interdiscip Plast Reconstr Surg DGPW. 2018;(7):Doc05. DOI: 10.3205/iprs000125.

13. Mirzaie M, Parsa A, Salehi M, Dahmardehei M, Moghadam $\mathrm{MH}$, Mirzaie N. Epidemiology of hand injuries in children presenting to an orthopedic trauma center in southeast of Iran. Arch Bone Jt Surg. 2014;2(3):225-31.

14. Capstick R, Giele H. Interventions for treating fingertip entrapment injuries in children. Cochrane Database Syst Rev. 2014;2014(4):CD009808. DOI: 10.1002/14651858.CD009808.pub2.

15. O'Donovan DA, Mehdi SY, Eadie PA. The role of Mepitel silicone net dressings in the management of fingertip injuries in children. J Hand Surg Br. 1999;24(6):727-30. doi: 10.1054/ jhsb.1999.0270.

16. Seymour N. Juxta-epiphysial fracture of the terminal phalanx of the finger. J Bone Joint Surg Br. 1966;48(2):347-9.

17. Krusche-Mandl I, Köttstorfer J, Thalhammer G, Aldrian S, Erhart J, Platzer P. Seymour fractures: retrospective analysis and therapeutic considerations. J Hand Surg Am. 2013;38(2):25864. DOI: 10.1016/j.jhsa.2012.11.015.

18. Abzug JM, Kozin SH. Seymour fractures. J Hand Surg Am. 2013;38(11):2267-70. DOI: 10.1016/j.jhsa.2013.08.104.

19. Lin JS, Popp JE, Balch Samora J. Treatment of acute Seymour fractures. J Pediatr Orthop. 2019;39(1):e23-7. DOI: 10.1097/ BPO.0000000000001275.

20. Al-Qattan MM. Phalangeal neck fractures in children: classification and outcome in 66 cases. J Hand Surg Br. 2001;26(2):11221. DOI: $10.1054 /$ jhsb.2000.0506.

21. Kattan AE, AlShomer F, Alhujayri AK, Alfowzan M, Murrad KA, Alsajjan H. A case series of pediatric Seymour fractures related to hoverboards: increasing trend with changing lifestyle. Int J Surg Case Rep. 2017;38:57-60. DOI: 10.1016/j.ijscr.2017.06.058.

22. Abzug JM, Ho CA, Ritzman TF, Brighton BK. Transphyseal fracture of the distal humerus. J Am Acad Orthop Surg. 2016;24(2):e39-44. DOI: 10.5435/JAAOS-D-15-00297.

23. Waters P, Beaty J, Kasser J. Elbow “TRASH” (The Radiographic Appearance Seemed Harmless) lesions. J Pediatr Orthop. 2010;30:S77-81. DOI: 10.1097/BPO.0b013e3181c18a9f.

24. Al-Qattan MM. Extra-articular transverse fractures of the base of the distal phalanx (Seymour's fracture) in children and adults. J Hand Surg Br. 2001;26(3):201-6. DOI: 10.1054/ jhsb.2000.0549.

25. Tan RE, Lim JX, Chong AK. Outcomes of phalangeal neck fractures in a pediatric population. J Hand Surg Am. 2020;45(9):880. e1-6. DOI: 10.1016/j.jhsa.2020.02.019.

26. Van Beek AL, Kassan MA, Adson MH, Dale V. Management of acute fingernail injuries. Hand Clin. 1990;6(1):23-35.

27. Klifto CR, Lavery JA, Gold HT, Milone MT, Karia R, Palusci V, Chu A. Pediatric fingertip injuries: association with child abuse. JHS GO. 2020;2(1):31-4. DOI: 10.1016/j.jhsg.2019.09.001. 